\title{
Can Magnetic Resonance Imaging Replace Mammography and Ultrasonography for the Detection of Breast Lesions?
}

\author{
Yeliz Yilmaz ${ }^{1}$, Gulten Sezgin Bener ${ }^{2}$, Kemal Atahan ${ }^{1}$, Nihan Acar ${ }^{1}$, Turan Acar ${ }^{1}$, Haldun Kar $^{1}$, Furkan \\ Tosun ${ }^{1}$, Melek Gokova ${ }^{1}$ \\ 1. General Surgery, İzmir Katip Celebi University, Atatürk Training and Research Hospital, İzmir, TUR 2. Radiology, \\ İzmir Katip Celebi University, Atatürk Training and Research Hospital, İzmir, TUR
}

Corresponding author: Yeliz Yilmaz, dryelizyilmaz@yahoo.com

\section{Abstract \\ Objective}

We aimed to evaluate the role of magnetic resonance imaging (MRI) in the visualization of breast lesions and to estimate whether MRI can be a reliable alternative to mammography (MG) and ultrasonography (USG) for this purpose.

\section{Materials and methods}

In this retrospective, single-center study, an analysis of medical files of 260 patients with breast masses as breast imaging reports and data system (BI-RADS) 4 and 5 at MRI was performed. The features of the breast lump, such as the side, location, multi foci or multicentricity, histopathological diagnosis, contrastenhancement characteristics, radiological, and pathological axillary involvement, were noted. Consistency between MRI-BIRADS and MG+USG-BIRADS, as well as the association between lesion characteristics, was sought.

\section{Results}

The agreement ratio between the BI-RADS categories of MRI and MG+USG was 0.654 while consistency between histopathological diagnosis and MRI BI-RADS category was 0.838 . The agreement between the BIRADS category of MG+USG and histopathological diagnosis was 0.819 . The consistency between MRI BIRADS and MG+USG BI-RADS increased remarkably with the advancement of age. Similarly, the consistency between MRI BI-RADS and histopathological diagnosis tends to increase with the advancement of age. Nonmass contrast enhancement yielded the highest agreement ratios between MRI BI-RADS and MG+USG BI-RADS, histopathological diagnosis and MRI BI-RADS, and histopathological diagnosis and MG+USG BI-RADS.

\section{Conclusion}

Received 04/14/2020 Review began 04/28/2020 Review ended 05/04/2020 Published 05/13/2020

() Copyright 2020

Yilmaz et al. This is an open access article distributed under the terms of the Creative Commons Attribution License CC-BY 4.0., which permits unrestricted use, distribution, and reproduction in any medium, provided the original author and source are credited.
Dynamic MRI is a useful and reliable method for imaging breast neoplasms. However, it is not devoid of disadvantages such as cost, attainability, and contrast use and it should be reserved as a problem-solving technique to be used in conjunction with conventional methods including MG and USG.

Categories: Radiology, General Surgery, Oncology

Keywords: magnetic resonance imaging, breast tumor, imaging, mammography, ultrasonography, bi-rads

\section{Introduction}

Breast cancer constitutes one of the most important health problems in women, and survival depends on the size of the tumor and the involvement of lymph nodes at the time of diagnosis. Imaging of the breast tissue aims to screen asymptomatic cases and to evaluate the symptomatic patients properly. Since early diagnosis is the most crucial prognostic parameter, screening modalities have gained significance [1].

Even though the primary screening modality is mammography (MG), the accuracy of this method is reduced remarkably in dense breast tissues in the pre-menopausal period and cases receiving hormone replacement treatment. Moreover, MG may fail to distinguish benign tumors from malignant lesions attributed to its low specificity [2-3].

Ultrasonography (USG) constitutes an alternative diagnostic tool, particularly for patients with obscure imaging features under MG. The conjunctive use of MG and USG may not be sufficient to identify and document the behavioral patterns, multicentricity, and planning of conservative surgery and distinguish a residual lesion from granulation tissue. In such circumstances, magnetic resonance imaging (MRI) can be a 
useful diagnostic measure [4-5]. Owing to the use of contrast agents, MRI became a highly sensitive imaging modality for the screening and diagnosis of breast tumors. The sensitivity and specificity of MRI were reported as $83 \%-99 \%$, and $87 \%-97 \%$, respectively [6-9].

The philosophy of MRI is based on the assessment of longitudinal relaxation time (T1), transverse relaxation time (T2), and hydrogen spin density of tissues under investigation. There has been controversy on whether a breast lesion with contrast enhancement on MRI should be evaluated with high spatial resolution or dynamic contrast views should be preferred to achieve higher sensitivity [10]. Currently, attributed to the development of the MRI gradient system and pulse sequences, the concomitant use of high spatial resolution and sufficient temporal resolution has been popularized [10].

Increased awareness and a better understanding of the pathologic features of breast tumors may be useful to interpret the MRIs. Large lesional size (>10 mm), ill-defined margin, and irregular outline are mostly consistent with malignancy. These correlate with the pathological features of a breast tumor, characterized by the rapid growth rate, large size, infiltrative growth pattern, invasion into stroma resulting in desmoplasia, and, hence, irregular outline and margin. An integrated evaluation of clinical, radiological, and pathological characteristics is mandatory to accomplish highly accurate diagnostic outcomes [11]. The morphology and enhancement kinetics are helpful for the recognition of malignant breast tumors. In addition to discrimination of malignant and benign lesions, MRI can provide advantages for the detection of axillary lymph node metastases and the identification of occult primary [12].

The most commonly used reporting and classification system is Breast Imaging Reporting and Data System (BI-RADS ${ }^{\circledR}$ ), which was used with high-resolution 3 T systems [13-14]. BI-RADS categorization has been employed for the breast lesions using the morphological features and time-signal contrast curves of the lump. Dynamic MRI is useful for the BI-RADS classification of the breast lesions and to foresee the histopathological diagnosis. Dynamic contrast-enhanced MRI constitutes an improved imaging modality with advanced accuracy for differentiation between benign and malignant lesions. Early enhancement is supposed to imply malignancy [15-16].

The present study aimed to evaluate the role of MRI in the visualization of breast lesions and to estimate whether MRI can be a reliable alternative to MG and USG for this purpose.

\section{Materials And Methods \\ Study design}

This retrospective, single-center study was carried out in the radiology department of a tertiary care center between January 2014 and July 2019. The approval of the local institutional review board was obtained before the study (date: 01/2020 - no:530). The study was conducted in compliance with the principles of the Helsinki Declaration.

The descriptive and radiologic information was extracted from the medical files of our hospital database. Patients with breast lesions who were classified as BI-RADS 4 and 5 on MRI views were included. The consistency of the pathological lesions with the lesion type, contrast enhancement patterns, and radiological features on MRI was investigated. The harmony between BI-RADS classification and pathological diagnosis, as well as the accuracy for the demonstration of the axillary metastasis using MRI, were assessed. Patients who underwent both MG+USG and MRI for breast lesions were included in this study. All MRI views were evaluated by the same radiologist.

Data were collected from the medical records of 260 patients (258 women, 2 men) with an average age of $50.88 \pm 11.61$ (range: 20 to 80 ).

\section{Outcome measures}

Baseline descriptions, such as age and gender, BI-RADS classifications as for MG+USG and MRI, features of the breast lump, such as the side, location, multi foci or multicentricity, histopathological diagnosis, contrast-enhancement characteristics, and radiological and pathological axillary involvement, were noted. The location of the lesion was classified as the upper outer quadrant, upper inner quadrant, lower outer quadrant, lower inner quadrant, and central. The side of the lesion was classified as right, left, or bilateral.

\section{Conventional diagnostic imaging}

All patients underwent digital MG (IMS Giotto, Italy) and targeted ultrasonographic examination of the affected breast and the ipsilateral axillary region. Ezu-MT28-S1 model (Hitachi Inc. Japan) and a $13 \mathrm{MHz}$ linear transducer were used to evaluate the breast lesions before MRI. These examinations were performed by our radiology department in our tertiary care center.

\section{MRI protocol}




\section{Cureus}

Magnetic resonance imaging examinations were carried out using a 1.5-T imaging uni (Signa, GE Healthcare, Chicago, Illinois). For the Signa scanner, the imaging parameters were as follows: 4.6/2.2; flip angle, $10^{\circ}$; a field of view (FOV), $34 \times 34 \mathrm{~cm}$; matrix, $320 \times 320$; section thickness, $2 \mathrm{~mm}$; and acquisition time, 75 seconds. Non-contrast T2 and T1-weighted images were obtained in the prone position using the breast coil. A $0.01 \mathrm{mmol} / \mathrm{kg}$ contrast agent was used for contrast imaging (gadoterate meglumine Dotarem图, Guerbet; gadobutrol: Gadovist国, Bayer Healthcare) at a rate of $2 \mathrm{~mL} / \mathrm{s}$, which was followed by a $20 \mathrm{~mL}$ saline flush at the rate of $2 \mathrm{~mL} / \mathrm{s}$ ). Dynamic contrast-enhanced images were obtained at minutes one, two, and six after contrast material injection. Post-processing manipulation included the production of standard subtraction and maximum-intensity-projection images (MIP). The images were transferred to a workstation (Advantage Windows, software version 4.0, GE Healthcare) for analysis.

\section{Results}

The descriptive features of our series are presented in Table 1. Our patient population $(n=260)$ comprised 258 women and two men. The average age was $50.88 \pm 11.61$ (range: 20 to 80 ). On MRI views, 92 lesions (35.4\%) were reported as suspicious while 168 lumps were identified as highly probably malignant. The lesions most commonly involved the left side $(n=132,50.8 \%)$, followed by right side $(n=113,43.5 \%)$ and bilateral involvement ( $\mathrm{n}=15,5.8 \%)$. The number of multifocal and multicentric lesions were 40 (15.4\%) and 35 (13.5\%), respectively.

\begin{tabular}{|c|c|c|c|}
\hline & & $\mathbf{n}$ & $\%$ \\
\hline \multirow{2}{*}{ Sex } & Male & 2 & 0.8 \\
\hline & Female & 258 & 99.2 \\
\hline \multirow{2}{*}{ MRI } & Suspicious & 92 & 35.4 \\
\hline & Highly probably malignant & 168 & 64.6 \\
\hline \multirow{3}{*}{ Site of lesion } & Right & 113 & 43.5 \\
\hline & Left & 132 & 50.8 \\
\hline & Bilateral & 15 & 5.8 \\
\hline \multirow{5}{*}{ Location of lesion } & Upper outer & 108 & 41.5 \\
\hline & Upper inner & 39 & 15.0 \\
\hline & Lower outer & 43 & 16.5 \\
\hline & Lower inner & 20 & 7.7 \\
\hline & Central & 50 & 19.2 \\
\hline \multirow{2}{*}{ Multi foci } & Yes & 40 & 15.4 \\
\hline & No & 220 & 84.6 \\
\hline \multirow{2}{*}{ Multicentricity } & Yes & 35 & 13.5 \\
\hline & No & 225 & 86.5 \\
\hline \multirow{7}{*}{ BI-RADS for MG+USG/MRI } & Additional investigation needed & 71 & 27.3 \\
\hline & Negative & 3 & 1.2 \\
\hline & Benign & 10 & 3.8 \\
\hline & Probably benign & 15 & 5.8 \\
\hline & Suspicious & 47 & 18.1 \\
\hline & Highly probably malignant & 78 & 30.0 \\
\hline & Proven malıgnancy & 36 & 13.8 \\
\hline \multirow{4}{*}{ Histopathology } & Benign & 42 & 16.2 \\
\hline & Premalignant & 207 & 79.6 \\
\hline & Malignant & 11 & 4.2 \\
\hline & Mass lesion with contrast $\mathrm{e}$ & 193 & 74.2 \\
\hline
\end{tabular}




\section{Cureus}

Lesion type

Contrast enhancement without mass lesion

62

23.8

Focus

5

2

Yes

Axillary involvement on MRI

No

Pathological axillary involvement

\section{TABLE 1: Descriptive data $(n=260)$}

MRI - magnetic resonance imaging; MG - mammography; USG - ultrasonography

BI-RADS $®:$ Breast Imaging Reporting and Data System

The assessment of breast MRI was performed by the same radiologist and the report included the indication for scan and clinical information in addition to the dose and type of contrast material administered to the patient. The image findings under foci were breast density, the amount of parenchymal background enhancement, and relevant findings such as axillary involvement and side and location of the lesion. Even though lymph node evaluation is not a particular aim of breast MRI, it can reveal unsuspected axillary nodal involvement. Each report was associated with a diagnostic category and recommendations. The most commonly used reporting and classification system is BI-RADS [13-14].

This system that provides the convenience of communication between clinic-radiology, as well as standardization of the studies, was offered by the American College of Radiology (ACR) in 1993 for the standardization of MG reporting terminology in the name BI-RADS [17]. This internationally accepted system was reviewed in 2003 with the addition of the MG+USG and MRI classification [18]. The BI-RADS diagnostic categories are demonstrated in Table 2.

\begin{tabular}{|l|l|}
\hline Category & Definition \\
\hline 1 & Incomplete, additional imaging evaluation is needed \\
2 & Negative, no abnormalities \\
3 & Benign findings \\
\hline 4 & Probably benign findings \\
\hline 5 & Suspected malignancy \\
\hline 6 & Highly suspected malignancy \\
\hline
\end{tabular}

\section{TABLE 2: BI-RADS diagnostic categories}

BI-RADS $®$ : Breast Imaging Reporting and Data System

Figures 1-2 demonstrate two benign sclerosing adenoma lesions, which were false-positively diagnosed as BI-RADS 5 at MRI. Thus, it must be remembered that despite its high sensitivity and acceptable specificity, a substantial rate of false positive and false negative results are likely particularly if MRI is used alone. 


\section{Cureus}
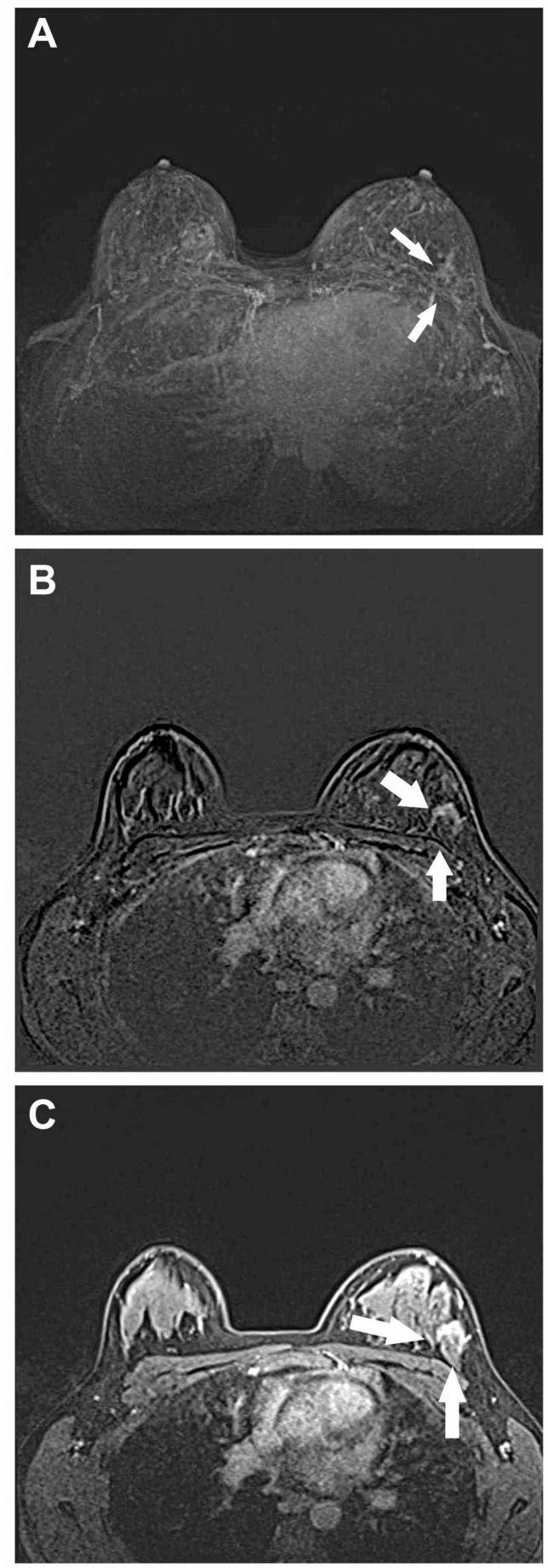

\section{FIGURE 1: Benign sclerosing adenoma lesion}

Nonmass enhancement in the upper outer quadrant in the left breast was detected on maximum intensity projection (a), on the subtracted image (b), and on the first passage of dynamic enhancement images (c). The lesion was consistent with sclerosing adenosis. 


\section{Cureus}
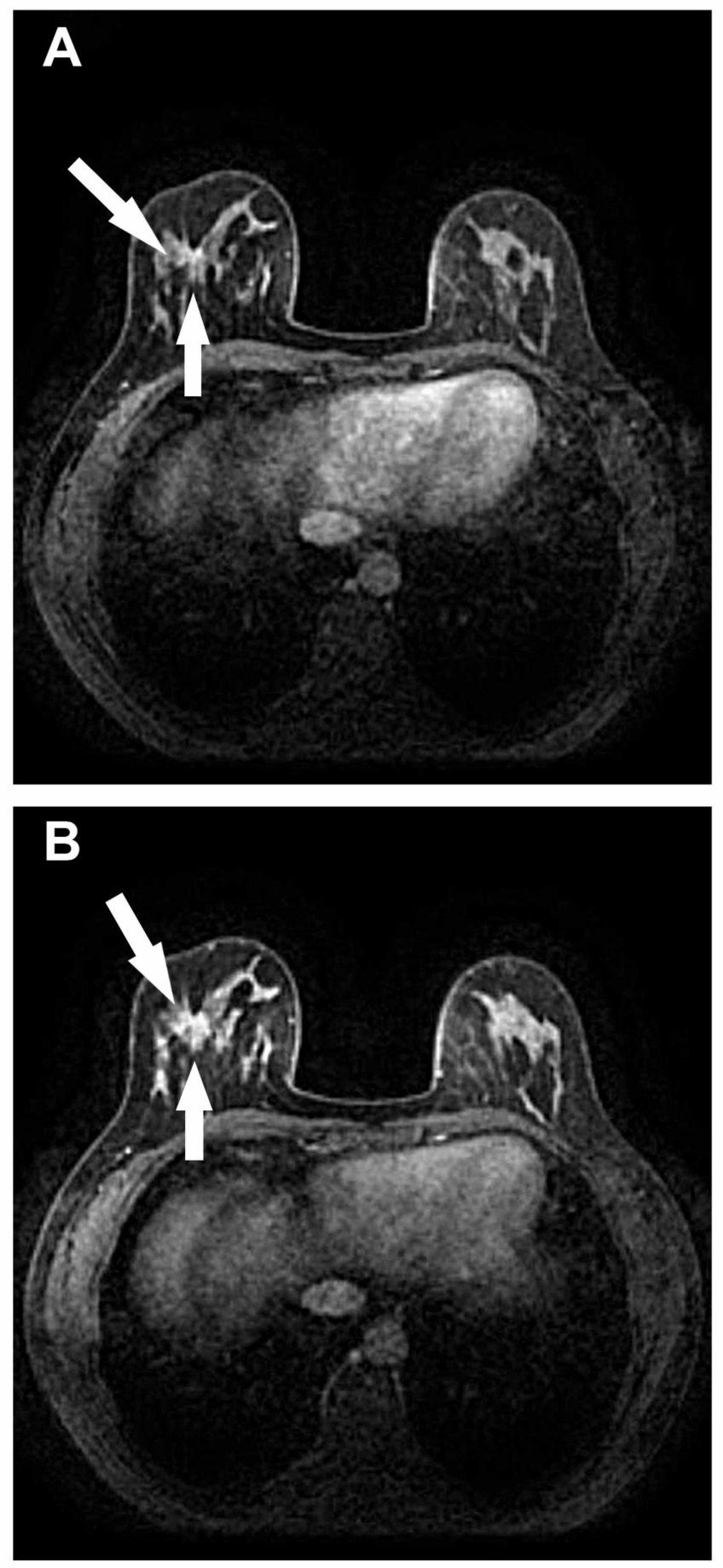

\section{FIGURE 2: Benign sclerosing adenoma lesion}

A structural distortion area was detected in the lower outer quadrant of the left breast on the second (a) and sixth passages of dynamic enhancement images (b). The lesion was sclerosing adenosis.

In Table 3, the agreement between the BI-RADS classification as for MRI and MG+USG and the relationship between the MRI BI-RADS category and other parameters under investigation were sought. The agreement ratio between the BI-RADS categories of MRI and MG+USG was 0.654 while consistency between histopathological diagnosis and MRI BI-RADS category was 0.838 . The agreement between the BI-RADS category of MG+USG and histopathological diagnosis was 0.819 . 


\section{Cureus}

\begin{tabular}{|c|c|c|c|c|}
\hline Variables & Agreement & Disagreement & Total & Agreement ratio \\
\hline MRI BI-RADS-MG+USG BIRADS & 170 & 90 & 260 & 0.654 \\
\hline MRI BI-RADS-Histopathological diagnosis & 218 & 42 & 260 & 0.838 \\
\hline MG+USG BI-RADS-Histopathological diagnosis & 213 & 47 & 260 & 0.819 \\
\hline MRI axillary lymph node-Histopathological diagnosis (specificity) & 180 & 80 & 260 & 0.692 \\
\hline MRI axillary lymph node-Histopathological diagnosis (sensitivity) & 176 & 84 & 260 & 0.677 \\
\hline
\end{tabular}

TABLE 3: Agreement ratios between MRI BI-RADS, MG + USG BI-RADS, axillary lymph node involvement on MRI and histopathological diagnosis

MRI: magnetic resonance imaging; MG: mammography, USG: ultrasonography; BI-RADS $®$ : Breast Imaging Reporting and Data System

Table 4 outlines the agreement ratios between MRI BI-RADS, MG+USG BI-RADS, and histopathological diagnosis as for various radiological characteristics on dynamic MRI views. We observed that the consistency between MRI BI-RADS and MG+USG BI-RADS increased remarkably with the advancement of age. Similarly, the consistency between MRI BI-RADS and histopathological diagnosis tends to increase with the advancement of age. Nonmass enhancement yielded the highest agreement ratios between MRI BI-RADS and MG+USG BI-RADS, histopathological diagnosis and MRI BI-RADS, and histopathological diagnosis and MG+USG BI-RADS

\begin{tabular}{|c|c|c|c|c|}
\hline MRI BI-RADS \& MG+USG BI-RADS & Agreement & Disagreement & Total & Agreement ratio \\
\hline \multicolumn{5}{|l|}{ Age groups } \\
\hline $0-20$ & 1 & 0 & 1 & 1.000 \\
\hline $21-40$ & 33 & 9 & 42 & 0.786 \\
\hline $41-60$ & 106 & 58 & 164 & 0.646 \\
\hline$>60$ & 30 & 23 & 53 & 0.566 \\
\hline \multicolumn{5}{|c|}{ MRI BI-RADS \& Histopathological Diagnosis } \\
\hline \multicolumn{5}{|l|}{ Age groups } \\
\hline $0-20$ & 1 & 0 & 1 & 1.000 \\
\hline $21-40$ & 39 & 3 & 42 & 0.923 \\
\hline $41-60$ & 139 & 25 & 164 & 0.820 \\
\hline$>60$ & 39 & 14 & 53 & 0.641 \\
\hline \multicolumn{5}{|c|}{ MG+USG BI-RADS \& Histopathological diagnosis } \\
\hline \multicolumn{5}{|l|}{ Age groups } \\
\hline $0-20$ & 1 & 0 & 1 & 1.000 \\
\hline $21-40$ & 34 & 8 & 42 & 0.810 \\
\hline $41-60$ & 135 & 29 & 164 & 0.823 \\
\hline$>60$ & 44 & 9 & 53 & 0.830 \\
\hline \multicolumn{5}{|l|}{ MRI BI-RADS \& MG+USG BI-RADS } \\
\hline \multicolumn{5}{|l|}{ Side } \\
\hline Right & 35 & 7 & 113 & 0.690 \\
\hline Left & 84 & 48 & 132 & 0.636 \\
\hline Bilateral & 8 & 7 & 15 & 0.533 \\
\hline
\end{tabular}




\section{Cureus}

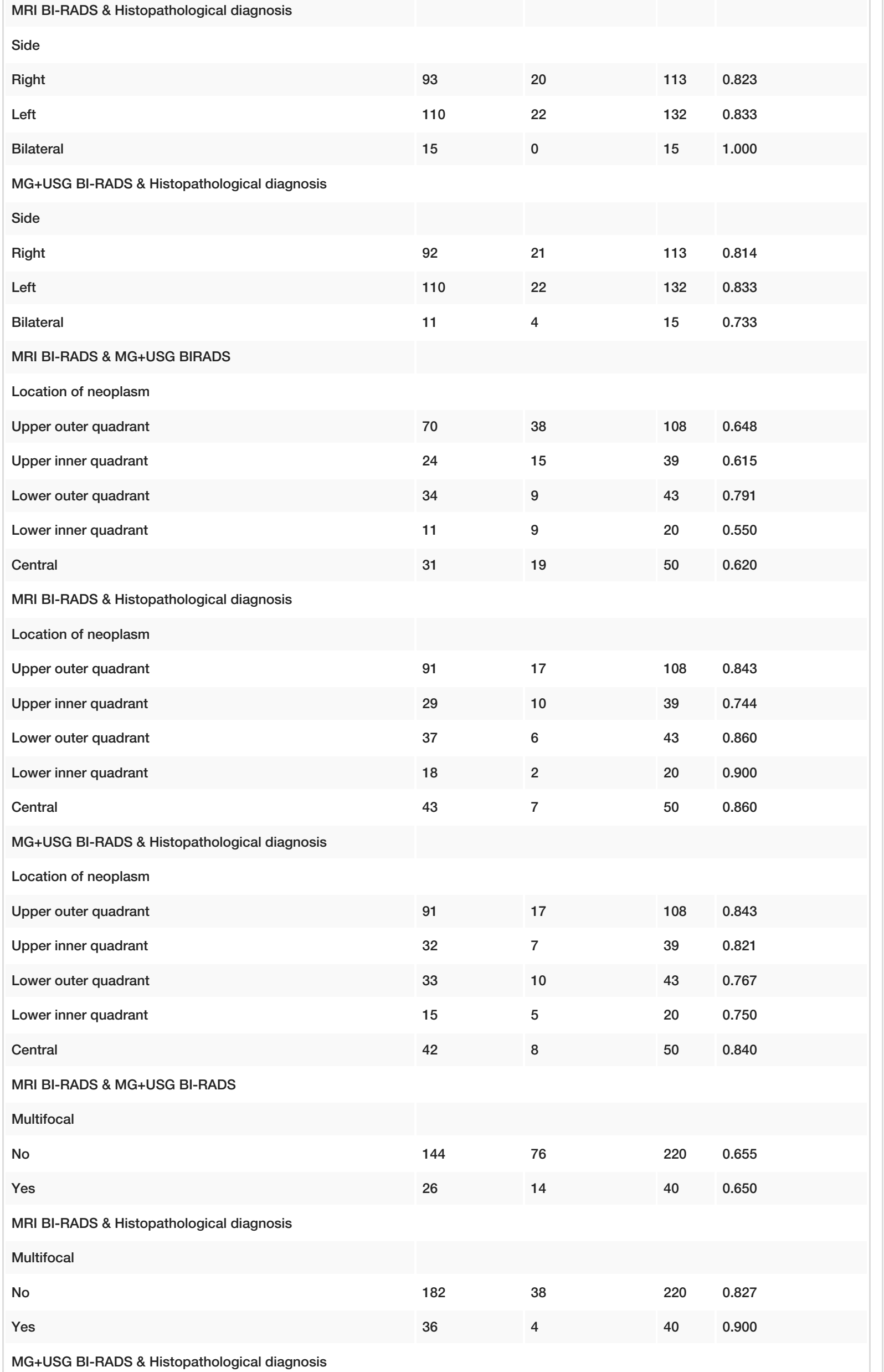




\section{Cureus}

Multifocal

No

MRI BI-RADS \& MG+USG BI-RADS

Multicentricity

No

MRI BI-RADS \& Histopathological diagnosis

Multicentricity

No

MG+USG BI-RADS \& Histopathological diagnosis

Multicentricity

No

MRI BI-RADS \& MG+USG BI-RADS

Type of lesion

Contrast enhanced mass

Contrast enhancement without mass

Focal

MRI BI-RADS \&Histopathological diagnosis

Type of lesion

\section{Contrast enhanced mass}

Contrast enhancement without mass

$\begin{array}{lll}71 & 193 & 0.632 \\ 16 & 62 & 0.742 \\ 1 & 2 & 0.500\end{array}$

MG+USG BI-RADS \&Histopathological diagnosis Type of lesion

Contrast enhanced mass

162

Contrast enhancement without mass

Focal

MRI BI-RADS \& MG+USG BI-RADS

Contrast enhancement

Fast

Slow

MRI BI-RADS \&Histopathological diagnosis

Contrast enhancement

Fast

Slow

MG+USG BI-RADS \&Histopathological diagnosis
$193 \quad 0.870$

$62 \quad 0.726$

$2 \quad 1.000$ 


\section{Cureus}

Contrast enhancement

Fast

Slow
112

101
29
130

130
0.862

0.777

TABLE 4: The agreement ratios between MRI BI-RADS, MG+USG BI-RADS and histopathological diagnosis as for various radiological characteristics on dynamic MRI views

MRI: magnetic resonance imaging; MG: mammography; USG: ultrasonography; BI-RADS $®$ : Breast Imaging Reporting and Data System

\section{Discussion}

We aimed to investigate the role of MRI in imaging breast lesions and in seeking the impacts of descriptive, clinical, and radiological variables on the agreement between MRI, MG+USG, and histopathological diagnosis. Our results demonstrated that the age of the patients, the location of the lesion, and enhancement characteristics might influence the ratio of agreement between MRI and MG+USG for the detection of breast neoplasms. Therefore, complementary use of imaging modalities together with an integrative analysis of clinical, pathological, and radiological data is mandatory to improve early and accurate diagnosis rates.

Magnetic resonance imaging is an important diagnostic measure in breast imaging, and dynamic contrastenhanced MRI is the backbone of any breast MRI protocol, with excellent sensitivity and good specificity for breast cancer diagnosis. It yields high-resolution morphological information and functional data about angioneogenesis as a tumor-specific feature [19].

The superiority of MRI is linked with its high sensitivity in tumor detection owing to the consistent contrast enhancement of breast cancer. In breast cancer, the enhancement of the tumor is always stronger than the normal breast tissue. Lack of enhancement reminds a benign lesion or normal tissue. Even pre-invasive lesions, such as lobular carcinoma in situ, showed stronger enhancement than normal glandular tissues. Malignant tumors of the breast frequently display an increased capillary network and increased permeability, and these factors contribute to the earlier and stronger contrast enhancement in breast malignancies [19]. Even though MRI has an important role in the detection of breast cancers, primarily in high-risk patients, one should be aware of the fact that false-negative MR findings do occur in a small percentage of cases. MG remains the main diagnostic technique for examination of the breasts. The MR imaging technique is of complementary value in better delineation of tumor size, in recognition of additional malignant lesions, and in mammographically difficult, dense breasts [20].

Reported high sensitivity (83\%-100\%) of MRI for breast cancer reinforced the presumption that nonenhancing lesions on MRI were benign and did not necessitate biopsy. However, it is known that not all malignant lesions display obvious contrast enhancement and enhancement was absent in up to $12 \%$ of malignant tumors [21].

The reasons for the misdiagnosis of these lesions were technical challenges, reader perception problems secondary to masking by intensely enhancing parenchyma, small lesion size, and diffuse growth patterns. However, estimation of the types of malignant lesions that are more likely to be missed at MRI needs to be investigated in further trials.

Small tumor size and diffuse parenchymal enhancement were likely the principal reasons for these falsenegative results. Although the overall sensitivity of breast MRI for cancer detection was high (96.8\%), it should be emphasized that a negative MRI should not influence the management of a lesion that appears to be of concern on physical examination, mammography, or ultrasound. MRI is complementary to, but is not a replacement for, other breast imaging techniques and should not be used as the sole imaging study because, as this study shows, a small number of cancers may not be visible at MRI [21].

The detection and characterization of malignant lesions are best performed using mammography and MRI. We suggest that making an MRI evaluation before proceeding to a histopathological diagnosis for suspected and indefinite breast lesions is the most preferable approach.

Our study possesses certain limitations such as retrospective design, selection bias, and lack of a control group. The possible impacts of biomechanical, social, environmental, and ethnic confounding factors must be remembered during the extrapolation of our results to larger populations.

Ideally, the radiologists dealing with breast imaging must be familiar and experienced with not only MRI but also they must be trained especially for conventional methods such as mammography and ultrasound. These methods are complementary to each other, rather than being alternatives. It must be remembered that 
integrative and multi-dimensional analysis of clinical, radiological, and pathological data is mandatory to reach the accurate diagnosis soon and to initiate the appropriate treatment without delay. Rather than using MRI instead of MG+USG or biopsy, MRI findings must be comparatively evaluated with other clinical and imaging findings.

\section{Conclusions}

Breast MRI is a sensitive imaging modality that remarkably improves screening in high-risk women. It has important functions in clinical diagnosis and staging, affecting patient management. Nevertheless, it is not a fully perfect diagnostic tool since some breast tumors may be missed and some benign lesions may be misdiagnosed as malignant. Therefore, clinical and other imaging findings from other modalities, such as mammography and USG, must be reviewed. These drawbacks must be known and shared with the patient before the performance of a breast MRI. In conclusion, dynamic MRI is a useful and reliable method for imaging breast neoplasms.

\section{Additional Information \\ Disclosures}

Human subjects: Consent was obtained by all participants in this study. Animal subjects: All authors have confirmed that this study did not involve animal subjects or tissue. Conflicts of interest: In compliance with the ICMJE uniform disclosure form, all authors declare the following: Payment/services info: All authors have declared that no financial support was received from any organization for the submitted work. Financial relationships: All authors have declared that they have no financial relationships at present or within the previous three years with any organizations that might have an interest in the submitted work. Other relationships: All authors have declared that there are no other relationships or activities that could appear to have influenced the submitted work.

\section{References}

1. Curpen BN, Sickles EA, Sollitto RA, Ominsky SH, Galvin HB, Frankel SD: The comparative value of mammographic screening for women 40-49 years old versus women 50-64 years old. AJR Am J Roentgenol. 1995, 164:1099-1103. 10.2214/ajr.164.5.7717212

2. Carney PA, Miglioretti DL, Yankaskas BC, et al.: Individual and combined effects of age, breast density, and hormone replacement therapy use on the accuracy of screening mammography. Ann Intern Med. 2003, 138:168-175.

3. Kerlikowske K, Grady D, Barclay J, Sickles EA, Ernster V: Likelihood ratios for modern screening mammography. Risk of breast cancer based on age and mammographic interpretation. JAMA. 1996, 276:3943.

4. Leung JWT: Screening mammography reduced morbidity of breast cancer treatment. AJR Am J Roentgenol. 2005, 184:1508-1509. 10.2214/ajr.184.5.01841508

5. Kolb TM, Lichy J, Newhouse JH: Comparison of the performance of screening mammography, physical examination and breast US and evaluation of factors that influence them: an analysis of 27,825 patient evaluations. Radiology. 2002, 225:165-175. 10.1148/radiol.2251011667

6. Kuhl CK, Mielcareck P, Klaschik S, Leutner C, Wardelmann E, Gieseke J, Schild HH: Dynamic breast MR imaging: are signal intensity time course data useful for differential diagnosis of enhancing lesions?. Radiology. 1999, 211:101-110. 10.1148/radiology.211.1.r99ap38101

7. Liu PF, Debatin JF, Caduff RF, Kacl G, Garzoli E, Krestin GP: İmproved diagnostic accuracy in dynamic contrast enhanced MRI of breast by combined quantitative and qualitative analysis. Br J Radiol. 1998, 71:501-509. 10.1259/bjr.71.845.9691895

8. Kinkel K, Helbich TH, Esserman LJ, Barclay J, Schwerin EH, Sickles EA, Hylton NM: Dynamic high spatialresolution MR imaging of suspicious breast lesions: diagnostic criteria and interobserver variability. AJR Am J Roentgenol. 2000, 175:35-43. 10.2214/ajr.175.1.1750035

9. Wiener JI, Schilling KJ, Adami C, Obuchowski NA: Assessment of suspected breast cancer by MRI: a prospective clinical trial using a combined kinetic and morphologic analysis. AJR Am Roentgenol. 2005, 184:878-886. 10.2214/ajr.184.3.01840878

10. Rauch DR, Edward H: How to optimize clinical breast MR imaging practices and techniques on your $1.5 \mathrm{~T}$ system. Radiographics. 2006, 26:1469-1484. 10.1148/rg.265055176

11. Tse GM, Chaiwun B, Wong KT, Yeung DK, Pang ALM, Tang APY, Cheung HS: Magnetic resonance imaging of breast lesions--a pathologic correlation. Breast Cancer Res Treat. 2007, 103:1-10. 10.1007/s10549-0069352-3

12. Morris EA, Schwartz LH, Dershaw DD, van Zee KJ, Abramson AF, Liberman L: MR imaging of the breast in patients with occult primary breast carcinoma. Radiology. 1997, 205:437-440. 10.1148/radiology.205.2.9356625

13. ACR BI-RADS Atlas ${ }^{\circledR}$ 5th edition. (2014). Accessed: August 14, 2014: https://www.acr.org/ClinicalResources/Reporting-and-Data-Systems/Bi-Rads.

14. Pinker-Domenig K, Bogner W, Gruber S, et al.: High resolution MRI of the breast at 3 T: which BI-RADS ${ }^{\circledR}$ descriptors are most strongly associated with the diagnosis of breast cancer?. Eur Radiol. 2012, 22:322-330. 10.1007/s00330-011-2256-6

15. Weinreb JC, Newstead G: MR imaging of the breast . Radiology. 1995, 196:593-610. 10.1148/radiology.196.3.7644617

16. Kerslake RW, Carleton PJ, Fox JN, et al.: Dynamic gradient-echo and fat-suppressed spin-echo contrastenhanced MRI of the breast. Clin Radiol. 1995, 50:440-454. 10.1016/S0009-9260(05)83159-9 


\section{Cureus}

17. Burnside ES, Sickles EA, Bassett LW, et al.: The ACR BI-RADS experience: learning from history . J Am Coll Radiol. 2009, 6:851-860. 10.1016/j.jacr.2009.07.023

18. Mann RM, Balleyguier C, Baltzer PA, et al.: Breast MRI: EUSOBI recommendations for women's information . Eur Radiol. 2015, 25:3669-3678. 10.1007/s00330-015-3807-z

19. Pinker K, Helbich TH, Morris EA: The potential of multiparametric MRI of the breast . Br J Radiol. 2017, 90:bjr20160715. 10.1259/bjr.20160715

20. Boetes C, Strijk SP, Holland R, Barentsz JO, Van Der Sluis RF, Ruijs JHJ: False-negative MR imaging of malignant breast tumors. Eur Radiol. 1997, 7:1231-1234. 10.1007/s003300050281

21. Shimauchi A, Jansen SA, Abe H, Jaskowiak N, Schmidt RA, Newstead GM: Breast cancers not detected at MRI: review of false-negative lesions. AJR Am J Roentgenol. 2010, 194:1674-1679. 10.2214/AJR.09.3568 